\title{
Una mujer ante el cáncer de mama en México*
}

\author{
Felicia Marie Knaul, $\mathrm{PhD}^{(1)}$
}

Coy una mujer, soy una mujer que vive con cáncer $\mathcal{O}$ de mama, y soy una mujer que vive con cáncer de mama en México. Vivir la enfermedad en México me ha permitido identificar la necesidad de acción colectiva por parte de las mujeres, y a favor de las mujeres, para enfrentar los desafíos de esta enfermedad en América Latina.

En este testimonio personal me centraré en compartir las lecciones que he aprendido a lo largo de un año de vivir con cáncer de mama. Estas lecciones conforman mi estrategia personal para enfrentar la enfermedad pero incluyen elementos para una llamada a favor de la acción colectiva.

Las lecciones se resumen en ocho puntos que versan alrededor de tres temas: la esfera personal y familiar; los elementos claves de mi tratamiento médico que me han dado valor y la visión de futuro que se centra en la canalización de mi experiencia en un programa para combatir la epidemia de cáncer de mama que está plagando a México y Latinoamérica. En cada uno de estos temas ofrezco algunas reflexiones sobre las implicaciones para la salud de las mujeres.

\section{Mi experiencia con el cáncer de mama hasta el momento $\neq, \S$}

Tuve mi primera mastografía "de rutina" en Cuernavaca, Morelos el 27 de octubre de 2007. El 6 de noviembre se confirmó el diagnóstico de cáncer de mama. Tenía 41 años de edad. Mi cáncer fue identificado en algún punto entre la etapa uno y la etapa dos temprana. Al momento de su detección, presentaba dos tumores malignos en el cuadrante superior izquierdo de la mama, de $2.5 \mathrm{~cm}$ y $1.3 \mathrm{~cm}$, y carcinoma ductal invasor substancial. Se localizaron algunas células cancerosas aisladas en un

\footnotetext{
₹ Describo mi situación médica desde la perspectiva de una persona que ha leído mucho sobre el tema, mantiene registros médicos cuidadosamente y trabaja en el sector salud, pero que no tiene entrenamiento formal en medicina.

$\S$ Para información sobre el criterio para el establecimiento de etapas y otra terminología a que se hace referencia en el texto, véase American Cancer Society $(2007)^{2}$ y Susan G. Komen for the Cure ${ }^{3}$ además de los sitios de internet en las notas a pie de página.
}

* Este ensayo retoma los elementos de "I am a woman who lives with breast cancer". Reproductive Health Matters 2008;16(32):I33-138.'

(I) Cáncer de mama: Tómatelo a pecho y Observatorio de la Salud. Instituto Carso de la Salud y Competitividad y Salud, Fundación Mexicana para la Salud. México, D.F. 
ganglio linfático, pero según los criterios actuales, los ganglios salieron sin invasión de cáncer.

Mis tumores eran (es importante usar el tiempo pretérito, puesto que ahora están fuera de mí, fijos en un bloque de láminas y parafinas de laboratorio y en una serie de cajitas de plástico) positivos a sobreexpresión de HER2, * así como a receptores de estrógeno y progesterona. Soy BRCA- $1 / 2^{\ddagger}$ negativa, y no hay historial de cáncer de mama en mi familia inmediata, hasta donde sabemos.

Me he sometido a tres intervenciones quirúrgicas en la mama. La primera cirugía fue la más atemorizante. Se extirpó un segmento de la mama izquierda y cinco ganglios linfáticos que presentaban un riesgo de cáncer invasor. Desafortunadamente, dado que uno de los márgenes continuó mostrando la presencia de cáncer invasor, se me tuvo que practicar una segunda cirugía, que fue realizada dos semanas después. Se extirpó otro segmento, en un intento de dejar los márgenes libres del cáncer. Desgraciadamente, esta segunda intervención no tuvo éxito, y un margen continuó mostrando infiltración. Mediante una IRM ${ }^{\S}$ se identificó, posteriormente, una masa adicional y relativamente grande en el cuadrante inferior derecho. Aunque resultó no-maligno, combinada con el margen invadido, esta situación hizo aconsejable la mastectomía, misma que se realizó el 5 de diciembre.

\footnotetext{
* HER2: Aproximadamente uno de cada cinco cánceres del seno contiene una cantidad muy elevada de una proteína llamada HER2/ neu. A los tumores con niveles aumentados de HER-2/neu se les conoce como "HER2-positivo". Normalmente son cánceres más agresivos pero responden a Herceptin. ${ }^{4,5}$

₹ BRCA 1/2 (Cáncer de Mama 1 y Cáncer de Mama 2) son genes (segmentos de $\mathrm{ADN}$ que son las unidades biológicas de la herencia) del humano asociados con un incremento significante en el riesgo de desarrollar cáncer de mama. Muchas de las que presentan cáncer de mama tienen familiares con antecedentes de la enfermedad y los científicos consideran que, en ocasiones, la vulnerabilidad a este cáncer se asocia a un componente genético (hereditario). Las mutaciones (variaciones genéticas hereditarias) de BRCA1 y BRCA 2 parecen explicar alrededor de 5\% de los cánceres de mama diagnosticados cada año en Estados Unidos. En general, las mujeres tienen alrededor de $12 \%$ de probabilidades (una de cada 8 ) de desarrollar cáncer de mama. Durante su vida, las mujeres con una mutación del BRCA1 o BRCA2 corren un riesgo de hasta $87 \%$ de desarrollar cáncer de mama. Las pruebas genéticas pueden detectar mutaciones en los genes BRCA1 y BRCA2. Durante su vida, las mujeres con estas mutaciones también corren un mayor riesgo de presentar cáncer de ovario: hasta $54 \%$ en el caso de mutación del BRCA1 y hasta $27 \%$ para la del BRCA2. ${ }^{6}$

§ IRM: Los exámenes con imágenes por resonancia magnética utilizan ondas de radio e imanes muy potentes en lugar de rayos X. Un material de contraste, llamado gadolinio, a menudo se inyecta en una vena para que las estructuras se muestren con más detalle., ${ }^{4,8}$
}

La mastectomía* fue, por mucho, la más dolorosa de las cirugías. Se hizo con un proceso de conservación de la piel y en el mismo momento, se insertó un expansor. El resultado es una cicatriz que se ve como un fruncimiento (o una sonrisa hacia abajo) que atraviesa el sitio donde estaba mi mama. Mi última, y menos difícil, intervención quirúrgica de 2007, fue para insertar un catéter en el lado derecho de mi tórax, para facilitar la infusión de la quimioterapia. El resultado es una cicatriz más pequeña y una pieza de metal en forma de botón que se ve, y se siente, como un timbre. Completé 16 dosis de quimioterapia (cuatro ciclos de FEC ${ }^{\ddagger}$ y 12 ciclos de Taxol\$) que duraron desde el 10 de enero hasta la tercera semana de junio del 2008 -casi seis meses.

No daré muchos detalles sobre los síntomas de la quimioterapia. En términos generales, pude manejarlos sorprendentemente bien y continuar con mis actividades en forma normal. Hubo días en los cuales, si no fuera por la calvicie, ni la que escribe, ni las personas a mi alrededor, hubiéramos sabido que estaba en tratamiento con quimioterapia.

Con la ayuda de una cantidad substancial de medicamentos, logré evitar la náusea y rara vez estuve cansada. Comencé a perder el cabello el 23 de enero de 2008, y hacia finales del mes estaba completamente calva. Este proceso continuó con casi todo el resto de mi vello corporal aunque felizmente, permaneció alguna cantidad de cejas y pestañas durante casi todo el tratamiento. A veces tuve dificultad para concentrarme y escribir -como si mi cerebro no fuera capaz de comunicarse con mis manos. El fenómeno que llaman "quimiocerebro" ha continuado," pero afortunadamente parece ser menos intenso en los últimos meses. Sin embargo, sigo confundiendo fechas, números y palabras.

* La mastectomía conlleva la extirpación de todo el tejido del seno, y algunas veces, junto con los tejidos adyacentes. ${ }^{4-9}$

₹ FEC: Quimioterapia que combina 5-fluorouracilo (5FU), Epirubicina y Cytoxina (Citoxina o Ciclofosfamida). ${ }^{10-14}$

$\S$ Taxol, o Paclitaxel, es un componente extraído de la corteza del árbol de tejo del Pacífico con actividad antitumoral obtenida vía semisintética del Taxus Baccata y se usa para tratar varios cánceres. ${ }^{17,18}$

\# Quimio-cerebro: La quimioterapia ha sido asociada con una trascendente disminución de la función cognitiva, aunque la mayoría de los pacientes retornan a la normalidad después de la terapia. Muchas mujeres que han sido sometidas a quimioterapia notan problemas cognitivos como un cambio en la concentración y la memoria, popularmente conocido como "quimio-cerebro". En los estudios se ha encontrado que el quimiocerebro es un efecto secundario del tratamiento, la mayoría de los síntomas a menudo desaparecen después de algunos años. ${ }^{4,17}$ 
He vivido también nueve meses de infusiones de Herceptin* y completaré el año de tratamiento en abril de 2009. Por tres años tomaré Zometa ${ }^{\ddagger}$ una vez cada seis meses para prevenir metástasis óseas, y por cinco años tomaré Tamoxifen. $\$$

En julio, con la primera dosis de Zometa y Herceptin, caí con bacteremia, ${ }^{\#}$ misma que se complicó con una fuerte reacción a la Zometa. Esto fue un golpe duro para mí, pues pensaba que todo iba a ser más sencillo después de la quimioterapia y al término del taxol. Después de una noche de fiebre alta, temblores que movieron la cama y bastante miedo, enfrenté otra hospitalización y una cirugía para extraer el catéter. Pero esta situación pasó rápidamente.

En septiembre tuve mi sexto viaje al quirófano para volver a colocar otro catéter, pues después de 16 rondas de quimioterapia, mis venas no estaban en buena condición para soportar las infusiones de Herceptin. Afortunadamente, este catéter sigue conmigo y está funcionando bien.

Estoy en el proceso de reconstrucción del seno. Debo admitir que esto se siente, tal vez, como tener una botella de agua caliente cosida a la pared de mi tórax. Hay un

\footnotetext{
* Herceptin (trastuzumabo) es una terapia dirigida, también llamada terapia biológica, y es un tipo más nuevo de tratamiento para el cáncer. Éste es un anticuerpo monoclonal (versión artificial de una proteína muy específica del sistema inmunitario). Se adjunta a la proteína promotora de crecimiento llamada HER2/ neu. La HER2/ neu se encuentra en pequeñas cantidades sobre la superficie de las células normales del seno y en grandes cantidades sobre las células cancerosas del seno. El cáncer de seno que contiene grandes niveles de esta proteína se denomina HER2/neu-positivo. Esta proteína acelera su crecimiento y propagación. Herceptin selectivamente se fija con gran afinidad en las células basales al dominio extracelular del receptor 2 de las proteínas HER 2 del factor de crecimiento epidérmico humano. Los estudios han demostrado que en mujeres con cáncer de mama positivo para HER2, en etapa precoz, este fármaco más la quimioterapia reducen el riesgo de que el cáncer regrese en $50 \%$. Es un medicamento para el tratamiento del cáncer de mama en pacientes cuyos tumores secretan la proteína HER2., 418,19

₹ Zometa (zoledronato) es un bifosfonato. Estos medicamentos pueden fortalecer los huesos que han sido debilitados al ser invadidos por las células del cáncer de seno y reducen el riesgo de fracturas. Además, los bifosfonatos pueden ayudar a prevenir el adelgazamiento de los huesos (osteoporosis) que se puede presentar a causa del tratamiento con inhibidores de la aromatasa (como se explica anteriormente) o de la menopausia temprana debida a la quimioterapia. ${ }^{20-23}$

$\S$ Tamoxifen (tamoxifeno) es un medicamento modulador selectivo de receptores de estrógenos que es utilizado como terapia hormonal para el tratamiento del cáncer de mama. Bloquea el efecto del estrógeno, el cual puede ayudar a las células cancerosas de la mama a sobrevivir y proliferar. ${ }^{24-26}$

\# Bacteriemia (bacteremia) es la presencia de bacterias en la sangre y
} suele estar asociada con una enfermedad grave. ${ }^{27}$ botón de metal, como otro timbre, colocado estratégicamente debajo de mi brazo izquierdo, justo donde debe pasar un sostén. Se inyecta agua a través del botón del "timbre" para hacer espacio, gradualmente, para un implante permanente. Debo prepararme para al menos una cirugía más para terminar la reconstrucción.

No quiero decir que ésta haya sido una experiencia indolora. Cuando tuve que enfrentar la mastectomía, pensé que me estaba mutilando. Cuando desperté de la mastectomía el dolor era tan severo que no podía respirar. Después, estaba aterrada por la quimioterapia. Tan aterrorizada, que inicialmente prefería afrontar el riesgo de morir que someterme al tratamiento. Sin embargo, y para mi sorpresa, he podido vivir con todo ello y crecer como persona.

\section{Cómo enfrento el cáncer}

Tengo una expresión que me ha acompañado desde los primeros días de mi quimioterapia: aunque aún no soy una sobreviviente, soy una ganadora. Pienso que soy una afortunada, no porque haya sobrevivido a la enfermedad-porque aún no he sobrevivido a ella, pero tengo una buena probabilidad de hacerlo- sino porque he ganado más de lo que he perdido.

En abril de 2008 cumplí 42 años de edad. Celebré mi cumpleaños sin mi mama izquierda, sin pelo, y sin mi ciclo menstrual, pero habiendo ganado una actitud positiva y una perspectiva de vida diferente, una capacidad de percibir la belleza a mi alrededor, y una tremenda sensación de orgullo en mí misma como mujer.

Quiero compartir la primera vez que en mi experiencia con el cáncer de mama me di cuenta de lo bien que me siento conmigo misma. Fue en una entrevista para un documental, a menos de tres semanas después de mi mastectomía, donde se me preguntó: “¿Qué es lo que ve cuando se mira en el espejo?". Al principio permanecí en silencio. En mi mente vi la imagen de mi cuerpo con una cicatriz grande donde antes tenía mi seno. Cuando me di cuenta de lo que sentía, comencé a sonreír, primero hacia mí misma, y luego hacia la cámara y dije, "veo a una mujer más fuerte de la que veía antes del cáncer."

Para lograr convivir con el cáncer y seguir viviendo, he desarrollado, como creo que la mayor parte de las pacientes hacen o deben hacer, estrategias para enfrentar la situación. Quiero compartir algunas de estas lecciones aprendidas de mi experiencia.

\section{Apreciar a tu familia; el cáncer los puede unir}

El cáncer de mama invade de manera muy dura no sólo tejidos, sino de manera figurada a todos los planos 
familiares. Comparto solamente algunos aspectos de la vivencia de la pareja y con las hijas. Cada día, desde mi diagnóstico, he pensado conscientemente lo afortunada que soy por tener dos hijas que llegaron a mi vida antes del cáncer, y que decidimos tenerlas cuando yo era capaz de hacerlo. Puedo ver hacia atrás y tener la certeza de que tomé las decisiones correctas en el tiempo correcto. Primero, dar a luz a Hannah a los 30 años, cuando acababa de terminar mi doctorado y a pesar de que estaba intentando ingresar al mundo del trabajo. $Y$ posteriormente, hacer llegar a nuestras vidas a Maha (Mariana Havivah) en 2004.

Una tarea difícil e importante era encontrar formas para compartir con Hannah y Maha el proceso de vivir mi cáncer. No se los escondo. Respondo lo que me preguntan, cuando me preguntan, pero solamente si preguntan. Les muestro lo que quieren ver, cuando quieren verlo, y si quieren verlo. Frecuentemente, esto significa leer libros y hacer dibujos. ${ }^{28-30}$

Creo que esta estrategia funcionó tanto para ellas como para mí, porque logramos una cercanía maravillosa. Después de las cirugías y los tratamientos, era la sonrisa de Maha de tres años de edad, lo que me quitaba los síntomas mucho más eficazmente que los medicamentos. En las pocas ocasiones en las que me sentía demasiado enferma para levantarme, la única persona que podía atenderme y hacerme sentir mejor era Hannah, de 11 años de edad, con un masaje y la receta de un té secreto que ella preparaba.

En el ámbito de la pareja, la lucha con el cáncer de mama nos hizo posible redescubrir la esencia de una relación y disfrutar momentos juntos. Nos dio la oportunidad, por varios meses, de separarnos de las pruebas del día a día de la vida, y centrarnos el uno en el otro. Me permitió la gran fortuna de un reencuentro con él y de recibir todo el cariño, ternura y respaldo que tanto había anhelado en los muchos años, durante los cuales él había estado trabajando tanto, que no podía permanecer mucho tiempo conmigo. A lo largo del año, conforme iba desapareciendo mi juventud y los elementos que me habían representado mi belleza física, él iba percibiendo algo diferente. Calva, mastectomizada y habiendo envejecido biológicamente 15 años, él me decía, "eres guapa" y me daba cuenta que así realmente me percibía. Mucho más importante ha sido que también se lo dice a las niñas, "Su mamá es muy guapa", espontáneamente y con mucha frecuencia. Así les estamos enseñando que la belleza de una persona es más que lo físico. En los momentos en que se me olvida, estas palabras: "eres guapa" me dan la seguridad de creer lo mismo.

\section{Apreciar el presente y el momento y vivir en ello}

Aprecio la belleza a mi alrededor, de una manera que nunca antes pude hacerlo. Comparo esta experiencia con la de una persona que se vuelve ciega y logra ser mucho más sensible al sonido. Ahora aprecio conscientemente los momentos cuando escucho el canto de los pájaros en la mañana, el olor de los florifundios, los momentos en los cuales mis hijas me hacen reír, los alimentos que verdaderamente disfruto al comer, el anaranjado intenso del arbusto de rosas y las puestas del sol que aprecié con Julio, mi esposo, desde la ventana del hospital, por sólo citar unos cuantos instantes. Ahora, registro todos estos momentos, en lugar de dejarlos pasar por alto, $\mathrm{y}$ permanecen conmigo.

\section{Llevar un sentido de normalidad en tu vida diaria}

Durante todo el proceso y aun en las peores quimioterapias, busqué mantener mis actividades de rutina. Acudo al trabajo todos los días, incluyendo la mayor parte de los días que tuve tratamiento. Mantengo una vida familiar que incluye jugar y nadar con las niñas. Salgo a cenar una vez a la semana con mi marido y viajo - para trabajar y para descansar- a muchas partes del mundo.

El ejercicio ha sido sorprendentemente importante en este proceso y he realizado entre 3 y 18 kilómetros en mi aparato elíptico casi todos los días. Desde mi cirugía, el ejercicio se ha convertido en mi forma de mostrarme que no estoy más enferma, sino cada día más sana. Es uno de los mejores "medicamentos" a mi disposición para controlar la náusea, el temor, la depresión, la ira y el aumento de peso que a menudo se asocian al tratamiento.

\section{Cuidar tu imagen personal}

Me esfuerzo en mantener una imagen personal y corporal buena, pero que esté en sintonía con una vida con cáncer. Yo creo que esta estrategia me ayudó mucho a vencer el miedo de verme calva. También creo que facilitó el proceso para mi familia, mis amigos, mis colegas y de las demás personas que me miraban sin pelo. $Y$ aunque esto pueda no ser terriblemente importante para muchas mujeres, lo era para mí.

El anterior estilo de vestir comenzó a no ser el adecuado debido a la mastectomía, mi pérdida de pelo, y las molestias de la quimioterapia. Mis costosas pelucas con cabellos largos de color castaño claro (hechas con el mío propio) nunca las sentí en armonía con mi cara, o con mi 
cabeza. Encontré mi camino en cubrirme la cabeza, colocar bufandas con moños y maquillar un poco más los ojos. Nunca usé una peluca -ni un solo día-y muchas veces, por lo segura que me sentía, algunas personas con las que me reuní no se dieron cuenta de que estaba calva.

\section{No esconderte ni dejar que el mundo te esconda}

He intentado evitar ser percibida con menos capacidades porque tengo cáncer. Por esto, tengo que dar las gracias a mis colegas, empleadores, y muchos otros por haber hecho posible administrar un "programa flexible" de trabajo acorde a vivir con mi tratamiento.

Aunque mis amigos y colegas han desempeñado un papel importante, también pienso que mi actitud lo fue. Nunca oculté mi cáncer, nunca pretendí ser capaz de trabajar como lo hacía antes, pero tampoco di a persona alguna la oportunidad de "no aceptarme" en el trabajo o ambientes públicos.

Tengo la impresión de que muchos pacientes con cáncer son discriminados, o se discriminan a sí mismos, y se retiran de la cotidianeidad de la vida diaria. También tengo la impresión de que esto sucede más con las mujeres, y que esto pudiera deberse a que son más fáciles de hacerse a un lado, o salirse del mundo laboral. Este tipo de discriminación -que emana tanto de la enfermedad como de la condición de género- se tiene que conquistar.

\section{Participar en la toma de decisiones sobre tu tratamiento}

Aunque podría centrarme en la excelencia técnica de mi tratamiento médico, lo que quiero enfatizar en esta parte de mi testimonio son otros aspectos más relacionados con el empoderamiento de la mujer y la paciente.

Creo que en las decisiones sobre el tratamiento, la participación del paciente, activa y bien informada, es clave para el éxito del mismo. Desde el momento en que decidí desempeñar un papel activo en las decisiones sobre mi tratamiento, comencé a sentirme mejor sobre él. Los momentos de mayor desesperación se presentaron muy al principio, cuando sentía que no sabía lo suficiente para decidir lo que debía hacer.

Esto no significa que haya sido fácil tomar las decisiones. Durante semanas agonicé sobre la decisión de la mastectomía, y elegí someterme a una cirugía adicional para extirpar más tejido, con la esperanza de salvar mi mama. Pero, cuando llegó el momento de someterme a la mastectomía total, tenía una certeza absoluta de que ésta era la decisión correcta, y creo que he sufrido mucho menos con su resultado debido a esta certeza.

\section{Informarte}

Para tomar decisiones, una paciente tiene que estar informada. No obstante que sea difícil localizar la información o entenderla, es clave buscar la información e informarse. Considero que es nuestro derecho y nuestra obligación, como mujeres y como pacientes, entender nuestra enfermedad y el tratamiento al cual estaremos sometidas.

Para mí, los momentos de mayor desesperación se presentaron muy al principio, cuando sentía que no sabía lo suficiente para decidir lo que debía hacer. En vez de generar más temor, la información me ayudó a comprender aquello por lo cual iba a pasar, por muy duro que fuera conocer o saber la verdad.

\section{Trabajar las pérdidas, especialmente las que se relacionan} con tu identidad de mujer

Considero también muy importante tomar en cuenta los efectos del tratamiento sobre nuestro sentir como mujeres. Haber tenido la oportunidad de reconstruir quirúrgicamente mi seno izquierdo fue clave para mi salud mental y aceptación de la mastectomía. Me ayudó a creer que los efectos del cáncer no iban a ser permanentes y me ayudó a pensar en cómo relacionarme con mis hijas cuando pasaran a través de su desarrollo mamario.

Conforme ha avanzado el proceso y el tratamiento, he tenido nuevas preocupaciones relacionadas con $\mathrm{mi}$ identidad como mujer. Durante la parte más pesada del tratamiento yo tenía presente la idea de que "eventualmente todo regresará a la normalidad". Ahora, debo enfrentar qué es lo que sí regresará y qué no.

La mayor parte de lo que no regresará a lo normal tiene que ver con las partes de mi cuerpo que definieron, durante la mayor parte de mi vida, mi sexualidad, y una parte importante de mi identidad como una mujer.

En primer lugar, sé que nunca más tendré sensibilidad en mi seno izquierdo. Pero además, hay que liderar con la menopausia prematura, violentamente inducida por la quimioterapia. A ello hay que sumar los síntomas de corto plazo, y conforme van pasando los meses, tengo que aceptar la probabilidad de que la menopausia sea permanente, aunque guardo esperanza de que se revierta.

La menopausia prematura ha sido una parte difícil del proceso de aprender a vivir con el cáncer y a la vez algo que en principio se trataba como una secuela un tanto secundaria. Ahora sé que ha sido y que sigue siendo para mí un golpe fuerte el hecho de haber envejecido 
-en términos de mi edad y salud "reproductiva"- el equivalente a 10-15 años en tan solo dos meses.

Poco se habla y poco se analiza este efecto del tratamiento. Sin embargo, representa una fuerte carga para las pacientes, carga que muchas tienen que ocultar al mundo y aun a ellas mismas.

No buscar regresar a lo que eras antes, celebrar lo que eres

Trabajo todos los días para tener una buena imagen de mí misma y para jamás anhelar la Felicia de antes. Trato de generar una nueva imagen acorde con como soy ahora, en vez de lamentar e intentar regresar a ser como era antes del cáncer. Es así que cuando mis hijas me preguntan cuándo tendré mi cabello tan largo como antes, les contesto que ahora estoy satisfecha con mi aspecto actual.

Al mismo tiempo, ya que estoy entrando en la fase final del tratamiento, o por lo menos la fase final de este primer encuentro con la enfermedad, tengo que ver hacia adelante y encontrar una vida sin cáncer, pero a la vez sin muchos elementos que definían mi vida y mi persona antes del cáncer. Eso también implica aprender a vivir con, pero no por ni a través de, el cáncer.

Es un cambio fundamental para un paciente, porque durante las fases agudas del tratamiento, nos toca vivir por el cáncer, pues las constantes y agobiantes invasiones del cuerpo hacen necesario dedicar gran parte de la vida a lidiar con los síntomas tanto físicos como emocionales.

Estoy en el proceso de aprender cómo encontrar formas y fórmulas de vivir sin lamentar lo que perdí con el tratamiento. Hay que procesar las pérdidas y el duelo para no hundirse en ello. Creo que implica buscar $\mathrm{y}$ encontrar nuevos proyectos y gustos, y celebrarlos. Visto de otra forma, de la forma correcta, es una oportunidad y un momento de redimensionar y ser creativo -oportunidades que pocas veces encuentra uno en la vida adulta por tener que enfrentar las presiones y el tren rutinario de la vida diaria.

\section{Buscar y celebrar lo que puedes hacer mejor que antes}

Busco lo que puedo hacer mejor, o cuando menos, en forma diferente, que como lo hacía antes del cáncer. El ejemplo más importante es la oportunidad de convertir mi experiencia personal con cáncer en un motor para generar un programa-Cáncer de mama: Tómatelo a Pecho. Esto ha proporcionado una nueva profundidad a mi trabajo como economista de la salud y me ha convertido en una persona con capacidad de abogar por los derechos de la salud, especialmente la de las mujeres.
La inspiración para crear el programa vino directamente de la sensación de frustración, dolor, pérdida, confusión, y sobre todo, del temor al tratamiento que experimentaría después de mi diagnóstico. Me di cuenta que todo sería aún peor si no tuviera o no hubiera tenido acceso al tratamiento.

Por primera vez en mis 20 años de carrera trabajando en salud en países en desarrollo, pude procesar internamente el terror experimentado por una paciente que no puede sufragar los gastos de su atención. Pensé en una mujer -diagnosticada de cáncer de mama a los 41 años de edad, con hijos pequeños, pero teniendo que elegir entre pagar por sus cuidados de salud y pagar a alguien que se hiciera cargo de sus hijos mientras está en el hospital. Estaba desgarrada y enferma por la injusticia de tener acceso a la atención médica, y que ella no tuviera la misma oportunidad.

Convertí varias de las lecciones que yo estaba aprendiendo, en materia para diseñar el proyecto que llegaría a ser Tómatelo a Pecho. Por lo mismo, la iniciativa se centra en promover información, investigación, innovación para una mejor detección temprana y conlleva un proyecto de fortalecimiento institucional.

"Tómatelo a Pecho" fue presentado al consejo directivo del Instituto Carso de la Salud el día anterior a mi mastectomía y recibió financiamiento clave para iniciar labores. Las actividades se iniciaron en México en marzo de 2007, y la iniciativa se está expandiendo a otras partes de Latinoamérica. ${ }^{31}$ El programa se basa en evidencia que va mucho más allá de mi propia experiencia. De hecho, los datos que empezamos a arrojar presentaron una clara justificación para el programa y demostraron una serie de mitos alrededor de la trayectoria de la enfermedad en México y en la región.

El cáncer de mama no es una enfermedad sólo del mundo desarrollado. Cerca de $45 \%$ de todos los casos de cáncer de mama, y 55\% de las muertes, ocurre en países de bajo ingreso. ${ }^{32}$ Además, no está restringido a mujeres de edad avanzada o posmenopáusicas y especialmente entre poblaciones de bajos ingresos está atacando a mujeres jóvenes. En 2006, el cáncer de mama fue la segunda causa de muerte en mujeres mexicanas entre 30 y 54 años de edad. ${ }^{33,34}$

En presencia de este gran desafío hay un mensaje alentador: el cáncer de mama es curable si se detecta tempranamente.

No obstante, en América Latina este mensaje aún no ha permeado ni a la paciente, ni al proveedor de salud básica, ni a la mayoría de las autoridades que crean las políticas. Además, muchas mujeres carecen de información y acceso a los estudios de detección temprana. Como resultado, pocos casos son detectados en etapas tempranas, cuando la probabilidad de curación 
es mucho más alta -en México esta cifra es solo de 5 a $10 \% .{ }^{35}$ Resolver estas barreras a los cuidados requiere acción -por parte de las mujeres, así como del sistema de salud.

En conclusión, me considero como la más afortunada de las pacientes. Tengo acceso a los mejores médicos y hospitales, a una familia que me apoya, a la información que necesito para entender mi padecimiento y su tratamiento y la oportunidad de llevar a cabo un programa para combatir la enfermedad. Estoy muy consciente de cuán afortunada soy.

También reconozco que sólo una parte de lo que relato de mi experiencia puede ser adoptada por cualquier mujer con cáncer de mama. Otra parte muy importante depende de la oportunidad de tener acceso a servicios de alta calidad mismo que no es la realidad para la mayoría de las latinoamericanas. La mayor parte de las pacientes enfrentan la enfermedad sin herramientas y además lo hacen muy tardíamente con reducidas posibilidades para vencerla. En gran parte por ello, alrededor de dos de cada tres muertes en México son evitables con detección temprana y tratamiento adecuado. ${ }^{36}$ Cambiar esta realidad es un desafío que tenemos que enfrentar colectivamente.

\section{Reconocimientos}

Quisiera agradecer a Julio Frenk por sus comentarios sobre un borrador anterior, así como por acompañarme a lo largo de mi experiencia con cáncer de mama. Agradezco también a Marge Berer por su trabajo de análisis y edición de una versión de este trabajo publicada en Reproductive Health Matters en noviembre de 2008; a Rebeca Moreno por sus comentarios y apoyo en la preparación de este documento; a Héctor Arreola-Ornelas, Héctor Gómez-Dantés, María Cecilia Gónzalez y Enrique Velázquez por su apoyo con la investigación; y a Jorge Merigo por su excelente apoyo con la traducción.

\section{Referencias}

I. Knaul FM. I am a woman who lives with breast cancer. Reproductive Health Matters 2008; 16(32): 133-138.

2.American Cancer Society. Breast cancer facts \& figures 2007-2008. Atlanta:American Cancer Society, 2007

3. Susan G. Komen for the Cure. Finding your way to wellness: Puget sound breast cancer information and resource guide. Seattle: Susan G. Komen for the Cure, 2007

4.American Cancer Society. Cáncer del seno (resumen). 2009. [consultado 2009 enero 18]. Disponible en: http://www.cancer.org/docroot/ESP/ content/ESP_4x_Cancer_del_seno_Resumen.asp
5. Burstein HJ.The Distinctive Nature of HER2-Positive Breast Cancers. N Engl J Med 2005; 353:1652.

6. Parmet S. Genética y cáncer de mama. JAMA [serie en internet] 2004. [consultado 2009 enero 18] Vol 292, No. 4, pp 522. Disponible en: http:// jama.ama-assn.org/cgi/data/292/4/522/DCI//

7. Enciclopedia Médica en español. Resonancia Magnética, definición y funcionamiento. Medline Plus. [Serie en Internet]. 2008. [consultado 2009 enero 22]. Disponible en: http://www.nlm.nih.gov/medlineplus/spanish/ ency/article/003335.htm

8. Smith RA.The Evolving Role of MRI in the Detection and Evaluation of Breast Cancer. N Engl J Med 2007; 356:1362.

9. Enciclopedia Médica en español. Mastectomía, definición y clasificaciones. Medline Plus. [Serie en Internet]. 2008. [consultado 2009 enero 22]. Disponible en: http://www.nlm.nih.gov/medlineplus/spanish/ ency/article/003335.htm

10. Instituto Nacional del Cáncer. 5-FU. Diccionario de cáncer. [Serie en Internet]. [consultado 2009 enero 22]. Disponible en: http://www.cancer. gov/templates/db_alpha.aspx!lang=spanish\&CdrID $=44752$

II. U.S. Food and Drug Administration, Center for Drug Evaluation and Research. FDA Oncology Tools Product Label Details in Conventional Order for capecitabine. Oncology Tools. [Serie en Internet]. [consultado 2009 enero 22]. Disponible en: http://www.accessdata.fda.gov/scripts/cder/ onctools/labels.cfm?GN=capecitabine

12. Instituto Nacional del Cáncer. 5-FU. Diccionario de cáncer. [Serie en Internet]. [consultado 2009 enero I8]. Disponible en: http://www.cancer. gov/templates/db_alpha.aspx?searchTxt=Epirubicina\&btnGo.x=0\&btnGo.y $=0$ \&sgroup $=$ Starts + with\&lang $=$ spanish

13. U.S. Food and Drug Administration . Epirubicin hydrochloride injection. Revised Package Insert. [consultado 2009 enero 22]. Disponible en: http:// www.fda.gov/cder/foi/label//999/50778lbl.pdf

14. U.S. Food and Drug Administration, Center for Drug Evaluation and Research. FDA Oncology Tools Product Label Details in Conventional Order for cyclophosphamide. [Serie en Internet]. [consultado 2009 enero 22].Disponible en: http://www.accessdata.fda.gov/scripts/cder/onctools/ labels.cfm? GN=cyclophosphamide

15. Instituto Nacional del Cáncer. Taxol. Diccionario de cáncer. [Serie en Internet]. [consultado 2009 enero 18]. Disponible en: http:// www.cancer.gov/dictionary/?search Txt=taxol\&btnGo.x=9\&btnGo. $y=4 \&$ sgroup $=$ Starts + with\&lang

16. Mead Johnson. TAXOL 7 (paclitaxel). FDA. [consultado 2009 enero

18]. Disponible en: http://www.fda.gov/cder/foi/label//998/20262s24lbl.pdf 17. Hayes DF. Follow-up of Patients with Early Breast Cancer. N Engl J Med 2007; 356:2505.

18. U.S. Food and Drug Administration, Center for Drug Evaluation and Research. FDA Oncology Tools Product Label Details in Conventional Order for trastuzumab. [Serie en Internet]. [consultado 2009 enero 22]. http://www.accessdata.fda.gov/scripts/cder/onctools/labels. $\mathrm{cfm}$ ? GN=Trastuzumab

19. Enciclopedia Médica en español. Cáncer de mama. Medline Plus. [Serie en Internet]. 2008. [consultado 2009 enero 18]. Disponible en: http://www.nlm.nih.gov/medlineplus/spanish/ency/article/0009/3. htm\#Tratamiento

20. U.S. Food and Drug Administration, Center for Drug Evaluation and Research. FDA Oncology Tools Product Label Details in Conventional Order for zeledronate. [Serie en Internet]. [consultado 2009 enero 22]. Disponible en: http://www.accessdata.fda.gov/scripts/cder/onctools/labels. cfm? $\mathrm{GN}=$ zoledronate

2I. Instituto Nacional del Cáncer. Zometa. Diccionario de cáncer. [Serie en Internet]. [consultado 2009 enero 18]. Disponible en: http://www.nci. nih.gov/Templates/db_alpha.aspx?CdrID=487473\&lang=spanish; 22. Web MD. Drugs \& Medications - Zometa IV [consultado 2009 enero 18]. Disponible en: http://www.webmd.com/drugs/drug-21757-Zometa+IV. aspx?drugid=21757\&drugname=Zometa+IV\&pagenumber $=4$ 
23. Novartis Oncology. Zometa. [consultado 2009 enero 18]. Disponible en: http://www.us.zometa.com/info/index.jsp

24. Enciclopedia Médica en español. Cáncer de mama. Medline Plus. [Serie en Internet]. 2008. [consultado 2009 enero 18]. Disponible en http://www. nlm.nih.gov/medlineplus/spanish/ency/article/0009/3.htm\#Tratamiento [consultado 2009 enero 18]

25. Instituto Nacional del Cáncer. Tamoxifeno. Hojas informativas. [Serie en Internet]. [consultado 2009 enero 18]. Disponible en: http://www. cancer.gov/espanol/cancer/hojas-informativas/tamoxifeno-respuestas 26. FDA. Profesional information brochure. Novaldex, Tamoxifen citrate. [consultado 2009 enero 18]. Disponible en: http://www.fda.gov/cder/foi/ label//998/I7970.pdf

27. Enciclopedia Médica en español. Septicemia. Medline Plus. [Serie en Internet]. 2008. [consultado 2009 enero 22]. Disponible en: http://www. nlm.nih.gov/medlineplus/spanish/ency/article/00I355.htm

28. Ackermann A. Our mom has cancer.Atlanta:American Cancer Society, 2001

29. Sutherland E. Mom and the polka-dot boo-boo: a gentle story explaining breast cancer to a young child. Atlanta:American Cancer Society, 2007
30. Heiney S, Hermann J, Bruss K, et al. Cancer in the family: helping children cope with a parent's illness. Atlanta:American Cancer Society, 200I

31. Presentación del proyecto "Tómatelo a Pecho". [Consultado 2009 junio 25]. Disponible en: http://www.tomateloapecho.org.mx/attachments/ File/documentos/Descripcion\%20del\%20programa.pdf; y http://www.salud. carso.org/prog_cancer0I.html

32. Porter P."Westernizing" women's risks? Breast cancer in lowerincome countries. N Engl J Med 2007;358(3):4

33. Knaul FM, Nigenda G, Lozano R,Arreola-Ornelas H, Langer A y Frenk J.The Breast Cancer Challenge in Latin America: evidence from Mexico. Reproductive Health Matters 2008; 16(32): I I3-1 23.

34. Knaul FM, Lozano R, Gómez-Dantés H, et al. Numeralia: México, cáncer de mama. México DF: Fundación Mexicana para la Salud/Instituto Carso de la Salud, 2008

35. Secretaría de Salud. Programa de acción: Cáncer de mama. México DF: Secretaría de Salud, 2002

36. Franco F, Lozano R, Villa B, et al. La Mortalidad en México, 2000-2004.

Muertes Evitables: magnitud, distribución y tendencias. México DF:

Dirección General de Información en Salud, Secretaría de Salud, 2006 